\title{
Magnetic Resonance Imaging in Breath-Hold Divers with Cerebral Decompression Sickness
}

\author{
Ryu Matsuo ${ }^{a}$ Masahiro Kamouchi ${ }^{b}$ Shuji Arakawa ${ }^{c}$ Yoshihiko Furuta $^{c}$ \\ Yuka Kanazawa $^{c}$ Takanari Kitazono ${ }^{a}$ \\ Departments of ${ }^{a}$ Medicine and Clinical Science, and ${ }^{b}$ Health Care Administration and \\ Management, Graduate School of Medical Sciences, Kyushu University, Fukuoka, and \\ ${ }^{\mathrm{C}}$ Department of Cerebrovascular Medicine, Japan Labour Health and Welfare \\ Organization, Kyushu Rosai Hospital, Kitakyushu, Japan
}

\section{Key Words}

Magnetic resonance imaging $\cdot$ Decompression illness $\cdot$ Blood-brain barrier · Vasogenic edema

\section{Abstract}

The mechanism of cerebral decompression sickness (DCS) is still unclear. We report 2 cases of breath-hold divers with cerebral DCS in whom magnetic resonance imaging (MRI) demonstrated distinctive characteristics. One case presented right hemiparesthesia, diplopia, and gait disturbance after breath-hold diving into the sea at a depth of $20 \mathrm{~m}$. Brain MRI with fluid-attenuated inversion recovery (FLAIR) sequence revealed multiple hyperintense lesions in the right frontal lobe, bilateral thalamus, pons, and right cerebellar hemisphere. The second case presented visual and gait disturbance after repetitive breath-hold diving into the sea. FLAIR imaging showed hyperintense areas in the bilateral occipito-parietal lobes. In both cases, diffusion-weighted imaging and apparent diffusion coefficient mapping revealed hyperintense areas in the lesions identified by FLAIR. Moreover, follow-up MRI showed attenuation of the FLAIR signal abnormalities. These findings are suggestive of transient hyperpermeability in the microvasculature as a possible cause of cerebral DCS.

(C) 2014 S. Karger AG, Basel

\section{Introduction}

A rapid reduction in environmental pressure causes decompression illness (DCI). DCI often develops in divers, compressed air workers, aviators, and astronauts [1], and is 
Matsuo et al.: Magnetic Resonance Imaging in Breath-Hold Divers with Cerebral Decompression Sickness

categorized into two pathological conditions, decompression sickness (DCS) and air gas embolism (AGE). Clinical manifestations are caused by regional gas nucleation in fatcontaining tissue in patients with DCS and invasion of gas into the systemic circulation in those with AGE.

DCI with neurological symptoms is estimated to occur in 2.7 per 10,000 divers [1]. Serious neurological disorders in DCI are likely caused by bubbles in the blood or tissue [1, 2]. Previous reports showed that multiple ischemic lesions could be detected using magnetic resonance imaging (MRI) in divers having neurological DCI with or without neurological symptoms $[3,4]$. Although brain imaging in neurological DCS has been reported by a few groups [5-7], the mechanisms of injury in DCS are still unclear. Here, we report 2 cases of DCS in whom MRI findings showed unique characteristics with an increase in the apparent diffusion coefficient (ADC) values.

\section{Case 1}

The patient was a free diver, who repeatedly dived to a depth of $20 \mathrm{~m}$. He felt chest pain, nausea, dizziness, and double vision after diving for several hours. On the following day, he was admitted to our hospital, and subsequent neurological examination revealed left abducens nerve palsy, right-sided sensory disturbance, dysmetria, and ataxic gait. Fluidattenuated inversion recovery (FLAIR) imaging, T2-weighted imaging (T2WI), and diffusionweighted imaging (DWI) showed multiple hyperintense lesions in the right frontal lobe, bilateral thalamus, pons, and right cerebellar hemisphere. The ADC value in these lesions was elevated (fig. 1). MR angiography and venography did not show any abnormalities. He was treated with hyperbaric oxygen therapy. After 2 weeks, his neurological symptoms improved and multiple lesions on MRI were attenuated (fig. 1).

\section{Case 2}

Another free diver repeatedly dived into the sea to a depth of $25-30 \mathrm{~m}$ for $6 \mathrm{~h}$. After diving, he felt dizziness and unsteady gait and therefore visited our hospital. Neurological examination showed left quadrant hemianopia and unstable gait. Brain MRI, including FLAIR, T2WI, and DWI, on admission demonstrated hyperintense areas in the bilateral occipito-parietal lobes. The ADC value in these lesions was increased (fig. 1). The patient then underwent hyperbaric oxygen therapy. After treatment for 3 weeks, his neurological symptoms disappeared and multiple hyperintense lesions on MRI were attenuated (fig. 1).

\section{Discussion}

We presented 2 cases with cerebral DCS, in whom the MRI findings, including DWI and $A D C$ mapping, were indicative of the presence of vasogenic edema. These findings indicate that vasogenic edema causes cerebral DCS and that MRI with DWI and ADC mapping is useful for the diagnosis of cerebral DCS.

MRI is more useful compared with other imaging modalities for the examination of patients with DCI [8]. Previous studies have reported that brain MRI revealed ischemic lesions in 6 out of 8 patients with AGE and focal hyperintensities in 2 out of 8 divers with DCS [8]. In patients with AGE, air gas occludes large arteries and causes territorial infarction. 
In contrast, MRI has a low sensitivity for the diagnosis of cerebral DCS not only in divers but also in aviators [9]. A neuropathological examination showed that DCS caused subtle and diffuse changes in the brain with no large areas of necrosis, including grossly dilated empty vessels in the white matter, small areas of focal necrosis in the gray matter, and diffusely distributed areas of vacuolation of the myelin around small blood vessels [10]. Quite recently, McGuire et al. [11] reported that U-2 pilots with occupational exposure to hypobaria showed a significant increase in lesion volume and number of white matter hyperintensities on MRI. Although these findings were observed in the chronic stage, white matter may be vulnerable to decompression.

In our 2 cases, DWI signal intensity was only slightly high, but the areas with FLAIR and T2 hyperintensity showed high ADC values. Moreover, the FLAIR, diffusion, and T2 signal abnormalities disappeared in follow-up MRI. Although ADC values increase in various pathological conditions [12], these findings mimicking posterior reversible encephalopathy syndrome support the idea that the underlying mechanism is vasogenic edema. During vasogenic edema, disruption of the blood-brain barrier (BBB) occurs and subsequently causes extravasation of plasma or red blood cells. Although posterior reversible encephalopathy syndrome is typically induced by specific pathological conditions such as accelerated hypertension, eclampsia, and autoimmune disorders, the present cases did not have these potential causes of vasogenic edema. Additionally, cerebral venous thrombosis could not be identified in these cases. There may be two possible ways in which decompression causes hyperpermeability in the microvasculature. Previous data showed that gas microbubbles generated from decompression and gas embolization led to endothelial dysfunction and mechanical injury of the BBB in experimental animals [13]. A recent report also suggested the possibility that gas microbubbles generated by decompression impaired endothelial function and caused capillary leak after inner ear DCS in a scuba diver [14]. Another possibility is that microbubbles or bubble-platelet aggregates in the postcapillary venules may cause stasis of capillary flow leading to disruption of the BBB.

The present cases raise the possibility that DCS and AGE can be differentiated using DWI and ADC mapping. The molecular diffusion in the affected brain tissue is facilitated in DCS but restricted in AGE. Although cerebrovascular dysfunction may occur frequently in divers without symptoms, such abnormalities are possibly detected using MRI. Recently, DCS has also been noticed in other circumstances than diving, for instance in aviators and astronauts. Therefore, further accumulation of cases is required to verify the underlying mechanisms in DCS.

\section{Disclosure Statement}

All authors report no conflicts of interest.

\section{References}

1 Vann RD, Butler FK, Mitchell SJ, Moon RE: Decompression illness. Lancet 2011;377:153-164.

2 Bove AA: Risk of decompression sickness with patent foramen ovale. Undersea Hyperb Med 1998;25:175178.

3 Kohshi K, Wong RM, Abe H, Katoh T, Okudera T, Mano Y: Neurological manifestations in Japanese ama divers. Undersea Hyperb Med 2005;32:11-20.

-4 Schwerzmann M, Seiler C, Lipp E, Guzman R, Lovblad KO, Kraus M, Kucher N: Relation between directly detected patent foramen ovale and ischemic brain lesions in sport divers. Ann Intern Med 2001;134:21-24. 
Matsuo et al.: Magnetic Resonance Imaging in Breath-Hold Divers with Cerebral Decompression Sickness

5 Warren LP Jr, Djang WT, Moon RE, Camporesi EM, Sallee DS, Anthony DC, Massey EW, Burger PC, Heinz ER: Neuroimaging of scuba diving injuries to the CNS. AJR Am J Roentgenol 1988;151:1003-1008.

-6 Tamaki H, Kohshi K, Sajima S, Takeyama J, Nakamura T, Ando H, Ishitake T: Repetitive breath-hold diving causes serious brain injury. Undersea Hyperb Med 2010;37:7-11.

7 Matsuo R, Arakawa S, Furuta Y, Kanazawa Y, Kamouchi M, Kitazono T: Neurological decompression illness in a Japanese breath-hold diver: a case report. Clin Neurol 2012;52:757-761.

8 Reuter M, Tetzlaff K, Hutzelmann A, Fritsch G, Steffens JC, Bettinghausen E, Heller M: MR imaging of the central nervous system in diving-related decompression illness. Acta Radiol 1997;38:940-944.

-9 Jersey SL, Jesinger RA, Palka P: Brain magnetic resonance imaging anomalies in U-2 pilots with neurological decompression sickness. Aviat Space Environ Med 2013;84:3-11.

10 Palmer AC, Calder IM, Yates PO: Cerebral vasculopathy in divers. Neuropathol Appl Neurobiol 1992;18:113124.

11 McGuire S, Sherman P, Profenna L, Grogan P, Sladky J, Brown A, Robinson A, Rowland L, Hong E, Patel B, Tate D, Kawano ES, Fox P, Kochunov P: White matter hyperintensities on MRI in high-altitude U-2 pilots. Neurology 2013;81:729-735.

$\checkmark 12$ Sener RN: Diffusion MRI: apparent diffusion coefficient (ADC) values in the normal brain and a classification of brain disorders based on ADC values. Comput Med Imaging Graph 2001;25:299-326.

-13 Nossum V, Hjelde A, Brubakk AO: Small amounts of venous gas embolism cause delayed impairment of endothelial function and increase polymorphonuclear neutrophil infiltration. Eur J Appl Physiol 2002;86:209-214.

14 Gempp E, Lacroix G, Cournac JM, Louge P: Severe capillary leak syndrome after inner ear decompression sickness in a recreational scuba diver. J Emerg Med 2013;45:70-73. 
Matsuo et al:: Magnetic Resonance Imaging in Breath-Hold Divers with Cerebral
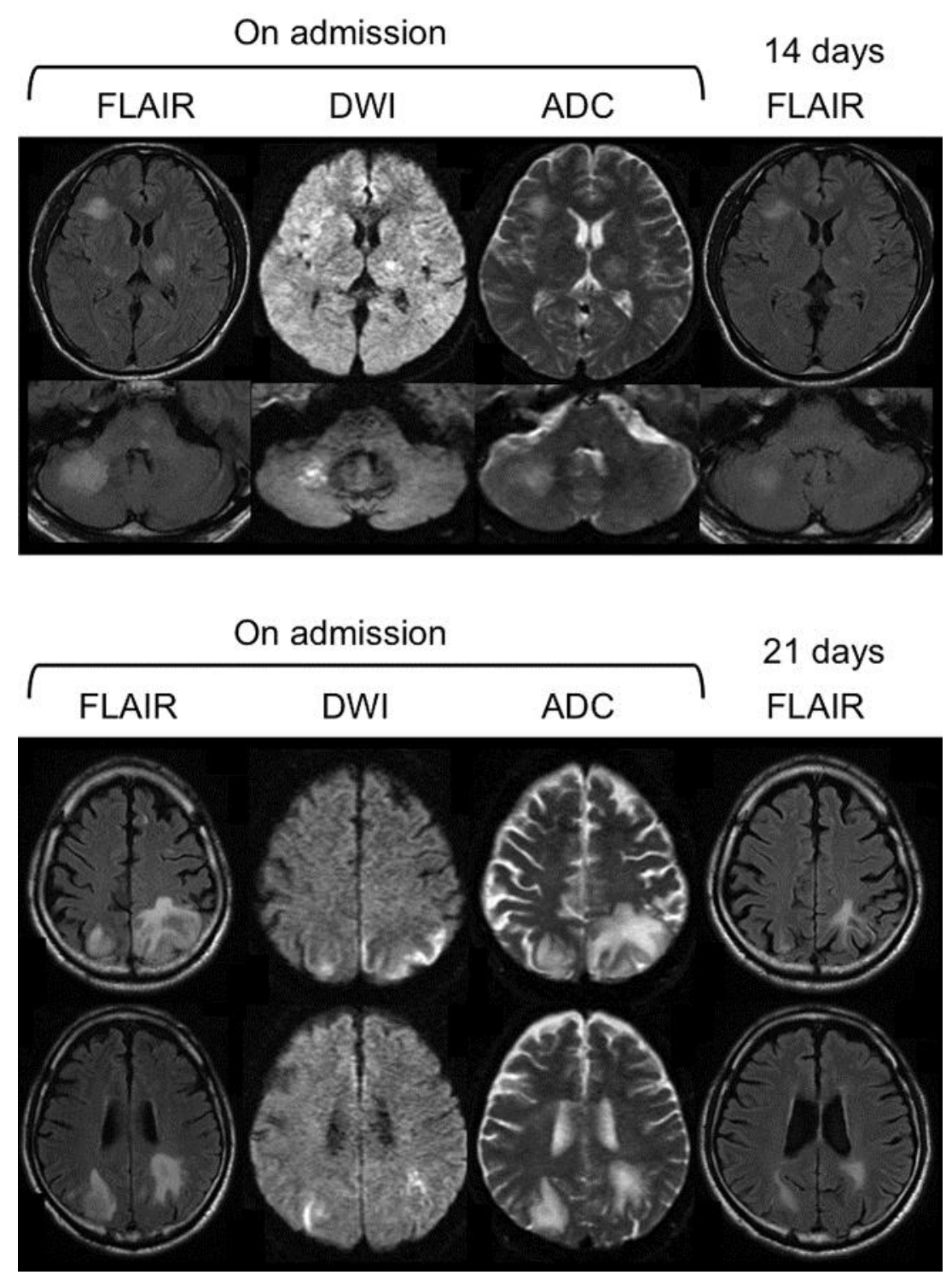

Fig. 1. FLAIR imaging, DWI, and ADC mapping. The upper panels show MRI findings in case 1 on admission and 14 days after onset. The lower panels show MRI findings in case 2 on admission and 21 days after onset. 